\title{
Cancer beyond genetics:
}

\section{On the practical implications of downward causation}

\author{
Sara Green ${ }^{{ }^{*}}$
}

\begin{abstract}
Discussions about reductionism and downward causation are often assumed to be primarily of interest to philosophers. Often, however, the question of whether multi-scale systems can be understood "bottom-up" has important practical implications for scientific inquiry. Cancer research, I argue, is one such example. While the focus on genetic factors has intensified with recent investments in cancer genomics, the importance of biomechanical factors within the tumor microenvironment is increasingly acknowledged. I suggest that role of solid-state tissue properties in tumor progression can be interpreted as a form of downward causation, understood as constraining relations between tissue-scale and micro-scale variables. Experimental demonstrations of these sort of influences reveal limitations of reductionist accounts and expose the dangers of what Wimsatt calls functional localization fallacies.

The latter relate to the common bias of downgrading factors that - as a practical necessity - are left out of scientific analysis. Any heuristic, experimental or theoretical, involves foregrounding some aspects while ignoring others, and the complexity of cancer leaves room for the co-existence of many different partial perspectives. These perspectives are not reducible to one another, but neither do they in this case make up a neatly integrated "causal mosaic" of different influences. At present, the picture of cancer research looks more like a fragmented cubist painting in need of a more balanced attention to difference-making factors at higher levels or scales.
\end{abstract}

\footnotetext{
${ }^{1}$ Section for History and Philosophy of Science, Department of Science Education, and Centre for Medical Science and Technology Studies, Department of Public Health,University of Copenhagen, DK. Email: sara.green@ind.ku.dk.

* I would like to thank Dan Brooks, James DiFrisco and Bill Wimsatt for organizing the workshop on Hierarchy and Levels of Organization and the KLI for hosting a great event. A version of the paper was presented at the Ghent-Brussels Seminars in Logic, History and Philosophy of Science where I benefitted particularly from comments from Leen de Vreese, Erik Weber, and Dingmar van Eck. Moreover, I received very helpful comments and suggestions from Bob Batterman, Anya Plutynski, Jim Woodward, Lauren Ross, Bill Bechtel, and the Philosophy of Science in Practice Group at UC San Diego. I also acknowledge The Carlsberg Foundation for research support (Semper Ardens grant CF17-0016).
} 
Final draft of a chapter to be published in D. S. Brooks, J. DiFrisco \& W. C. Wimsatt (Eds.), Biological Levels:

Composition, Scale and Evolution in Complex Systems. MIT Press.

\title{
1. Introduction
}

\author{
Cancer is no more a disease of cells than a traffic jam is a disease of cars. A \\ lifetime study of the internal combustion engine would not help anyone \\ understand our traffic problems (Smithers, 1962).
}

Debates on the characteristics of cancer are as tangled today as they were in the beginning of the $20^{\text {th }}$ Century (Baker, 2012; Plimmer, 1903). The question about the appropriate level of analysis is perhaps even more pressing in an era of large-scale investments in cancer genomics. Whereas some praise the potentials of genomics for understanding, stratifying, and treating different cancer types, others call for more attention to higher-level dynamics and organization of tissues. At stake are not only theoretical controversies but also issues with practical implications for experimental research and therapeutic interventions.

From a biological perspective, physics is often seen as a discipline aiming for reductionist (or fundamental) explanations. I challenge this view, and reductionism more generally, by focusing on the role of biomechanical features within the Tumor Microenvironment (TME) for cancer development (Laplane et al., 2018). I show how solid-state tissue properties in tumor progression supports the importance of macroscale features of living systems. Moreover, I suggest that biomechanical constraints can shed light on the controversial notion of 'downward causation' that is often used to distinguish between reductionist and anti-reductionist approaches in philosophy of biology (Campbell, 1974; Mossio et al., 2013; Wimsatt, 1994). An examination of downward causation in the context of cancer is of relevance also to broader questions about the explanatory scope of genetics.

Before we begin the analysis, it should be noted that "biological levels" are not unambiguously delineated (McGivern, 2008; Potochnik \& McGill, 2012). Levels can be defined spatially, functionally, or temporally (DiFrisco, 2017), and the notion can be sensitive to circumstances of its use such as different disciplinary contexts (Brooks \& Eronen, 2018; Wimsatt, 1972). In the following, I use the term "level" when referring explicitly to part-whole relations in organisms, such as genomes, cells, or tissues (see also Kaiser, 2015; Love, this issue; Wimsatt, 2007). However, the proposed view does not 
Final draft of a chapter to be published in D. S. Brooks, J. DiFrisco \& W. C. Wimsatt (Eds.), Biological Levels:

Composition, Scale and Evolution in Complex Systems. MIT Press.

depend on any particular specification of levels. I prefer the term scale when more broadly discussing the influence of macroscale features, which also has implications for physics (Batterman, 2012).

I begin with some background for the discussion of whether a gene-centric view on cancer is adequate (Sections 2 and 3). I then examine studies emphasizing the importance of macroscale biophysical features of the TME (Section 4). I argue that influences of physical features such as matrix stiffness can be interpreted as a form of downward causation, understood as constraining relations that can be mathematically interpreted as boundary conditions (Section 5). Sections 6 and 7 discuss implications for philosophical debates on reductionism and reflect on the possibility of a unified multi-scale approach to cancer. Section 8 offers a summary concluding remarks.

\section{Background for the debate}

The dominant view of cancer presented in almost all cancer biology textbooks is that carcinogenesis is driven by genetic instability in terms of somatic mutations that alter cell signaling pathways. Historically, this view has been called the Somatic Mutation Theory (Vaux, 2011) or the oncogene paradigm (Plutynski, 2018b). Cancer researchers today recognize that multiple factors influence the proliferation of cancer cells (Weinberg, 2007; Hanahan \& Weinberg, 2011), but most research efforts still focus on genetic factors and molecular pathways as targets of intervention and explanation. Recently, investigation of genetic factors has intensified through large-scale projects such as The Cancer Genome Atlas (TCGA, 2005-2016), which utilized automated sequencing tools and algorithms to identify genetic variants associated with different tumor types. Identification of cancer biomarkers are not only expected to increase our understanding of the diversity of cancer types but also to allow for personalized therapies (Tomczak et al., 2015).

In contrast to the optimism concerning new potentials of cancer genomics, some see investments in large-scale genome projects as "one more misstep in the war on cancer" (Miklos, 2005). Despite decades of heavy investment in research on genetic markers and molecular pathways involved in cancer development, results in terms of improved understanding and clinical control have so far been disappointing (Lazebnik, 2002; 2010; Prasad et al., 2016). Some have questioned whether "cancer 
Final draft of a chapter to be published in D. S. Brooks, J. DiFrisco \& W. C. Wimsatt (Eds.), Biological Levels: Composition, Scale and Evolution in Complex Systems. MIT Press.

cells", understood as entities with distinct molecular properties, can be meaningfully delineated at all (Soto \& Sonnenschein, 2011). The skepticism is motivated by a set of difficulties in distinguishing between mutational signatures in healthy and 'cancerous' cells, and between malignant and benign tumors (Baker, 2013; Hanahan \& Weinberg, 2011). Accordingly, some have argued that cancer is a disease that has to be characterized at higher scales or levels.

The importance of higher-level features for understanding cancer has been taken up by other philosophers, specifically in discussions of the Tissue Organization Field Theory (TOFT) (Sonnenschein \& Soto, 1999; 2011). This approach characterizes cancer as a failure of tissue organization. Philosophical debates have centered on the extent to which the frameworks of SMT and TOFT can be combined (Bedessem \& Ruphy, 2015; 2017), or whether these are theoretically incompatible due to conflicting ontological assumptions (Baker, 2013; Bizzarri \& Cucina, 2016; Montévil \& Pocheville, 2017). The debate provides intriguing insights into the implications of different ontological assumptions for our understanding of cancer. However, since the SMT and TOFT can be viewed as two extremes in a continuum of approaches to cancer, I shall focus more broadly on the role of the TME (see also Plutynski, 2018a).

The TME concept can encompass different experimental and theoretical approaches to cancer that go beyond TOFT (Laplane et al., 2018). The TME concept also has a long and diverse history, as it has been highlighted by immunologists and researchers focusing on angiogenesis since the 1970s (e.g., Folkman, 1971; Witz 2009). ${ }^{2}$ Central to emphasis on the TME is, however, that the environment of the tumor cells influences or constrains the possibilities of cell growth and proliferation. Similar insights have been stressed by developmental biologists that have experimentally demonstrated the influence of properties of the extracellular matrix (ECM) on development of breast cancer (Lochter \& Bissell, 1995). The framework of TME allows researchers to acknowledge top-down influences without denying the importance of genetic causation (Malaterre, 2011). Yet, as we shall see in the following, research on the role of the TME does challenge the explanatory scope of genetic approaches to cancer.

\footnotetext{
${ }^{2}$ Angiogenesis is a physiological process where new blood vessels are formed through branching from pre-existing vessels. Blood supply is often a bottleneck in tumor development and angiogenesis has recently become a target in cancer treatments.
} 
Final draft of a chapter to be published in D. S. Brooks, J. DiFrisco \& W. C. Wimsatt (Eds.), Biological Levels:

Composition, Scale and Evolution in Complex Systems. MIT Press.

\section{Postgenomic puzzles and recombination experiments}

The call for a higher-level perspective on cancer has a long history in biology but is currently also motivated by what Baker (2013) calls "postgenomic puzzles". The term refers to results of tumor sequencing that reveal unexpected complexity.

Efforts to understand cancer in terms of molecular mechanisms have been motivated by successful identification of genetic difference-makers from the 1970s and onwards. Examples are the tumor suppressor gene P53 and BRCA-markers used in risk analysis for breast cancer. Technologies such as Genome-Wide Association Studies have further resulted in the identification of a vast number of genetic factors and molecular pathways associated with cancer development. The genetic heterogeneity of tumors may even be so vast that each cancer is different. A widely cited quote from the US National Institute of Health with the launching of TCGA reads as follows:

Cancer is a disease of the genome and as more is learned about cancer tumors, the more we are finding that each tumor has its own set of genetic changes. Understanding the genetic changes that are in cancer cells is leading to more effective treatment strategies that are tailored to the genetic profile of each patient's cancer. ${ }^{3}$

While such quotes frame tumor heterogeneity is as a potential for individualize treatments, others interpret such discoveries as a severe challenge to the idea of associating cancer types with stable genetic markers.

Many tumors have been found to have heterogeneous mutational patterns. A few mutations are highly frequent in many cancer types, but a long tail (graphically speaking) of diverse mutations have been discovered both between and within tumors. Mutational signatures have been found to vary when biopsies are taken at different spatial locations in the tumor or within the same location over time

\footnotetext{
${ }^{3}$ The Cancer Genome Atlas Program: https://cancergenome.nih.gov/cancergenomics/impact, accessed 30/7-2018.
} 
Final draft of a chapter to be published in D. S. Brooks, J. DiFrisco \& W. C. Wimsatt (Eds.), Biological Levels: Composition, Scale and Evolution in Complex Systems. MIT Press.

(Blanchard \& Strand, 2017). Even more puzzling is that some tumors have normal mutation rates (Greenman et al., 2007; Qiu et al., 2008), and many cells with genetic lesions do not develop into tumors (Bissel \& Hines, 2011). Such results have engendered skepticism about whether cancer development can be captured in simple mechanistic models at the scale of genetic or molecular interactions. Similar cancer phenotypes can be realized through multiple genetic and molecular pathways, suggesting that it may be useful to search for higher-scale influences that bring clarity to the diverse influences.

Stromal components such as fibroblasts, immune cells, and the extracellular matrix, can make up to $80 \%$ of a tumor (Plutynski, 2018a, p. 25). Whereas much genetic research has focused on molecular changes within epithelial cells and their resulting behavior, the following shows how recombination experiments shift the focus to the structural role of connective tissues. In recombination experiments, cells are transplanted to new host organisms (or new TMEs). Recombination experiments allow for observation of the effects of cell development in response to experimental interventions where epithelial cells or the surrounding tissue are exposed to a carcinogen. Such experiments draw attention to how philosophers should not only pay attention to how scientists represent levels, but also how they manipulate or intervene on them (see also Love, this issue; Woodward, this issue).

Recombination experiments conducted in already in the 1950s suggested that carcinogen-induced "cancer cells" can be normalized if transplanted into normal tissue. ${ }^{4}$ Similarly, neoplastic induction was observed when normal cells were inserted in carcinogenous tissue. From the 1980s, systematic experiments were conducted of how features at different scales influenced development and carcinogenesis. As a consequence, Bissell and colleagues challenged the view that intercellular structures merely constituted "passive conditions" or "housekeeping functions" for the maintenance of cells (Plutynski, 2018b).

Bissell's group showed that tissue-specific traits of relevance to the development of cancer were often lost in 2D cultures (petri dishes with a single layer of cells). For instance, experiments demonstrated

\footnotetext{
${ }^{4}$ For a comprehensive review of important experiments, see (Baker, 2012; Bizzarri \& Cucina, 2014).
} 
Final draft of a chapter to be published in D. S. Brooks, J. DiFrisco \& W. C. Wimsatt (Eds.), Biological Levels: Composition, Scale and Evolution in Complex Systems. MIT Press.

that the fate of cells with virus-activated oncogenes depended on whether the cells were cultured in a petri dish or left in a chick embryo (Dolberg \& Bissell, 1984; Stoker et al., 1990). If the cells were removed from the embryo and cultured in a petri dish, they displayed the transformed phenotype within less than a day. However, if left in the embryo, the cells developed normally until much later. This suggests that the host environment plays an important role in buffering or dampening the effects of genetic lesions. More generally, the results suggest that the features of cancer cells are highly dependent on a context of reciprocal interactions with the microenvironment (see also Nelson \& Bissell, 2005; 2006).

Other historically important experiments were conducted by Ana Soto, Carlos Sonnenschein, and colleagues. They transplanted tumor cells from a donor mouse to normal tissue in mice hosts of different ages and hormonal stages (Maffini et al., 2005). If genetic instructions determined cell growth, one would expect the hosts to develop the same rate of tumors. Instead, they observed that tumor incidence varied among the hosts, and that in some cases no tumors formed at all. Similarly, from a reductionist perspective, replacement of healthy fat pads with pre-cultured tumor cells would be expected to result in tumor development. Yet, they observed normal development of tissue structures in all hosts. These experiments support the view that neoplastic cells can be normalized, depending on environmental conditions.

The cellular environment can also direct cell fates in the opposite direction: normal cells can undergo neoplastic induction if they are transplanted to tissue environments with carcinoma-exposed stroma. Maffini et al. (2004) investigated whether cancer originates in the stroma tissue or (bottom-up) with genetic mutations in epithelial cells. They surgically separated the stroma and epithelium such that each of these could be exposed to a chemical carcinogen. Interestingly, they observed that neoplastic transformation occurs only when the stroma had been exposed to the carcinogen. Similar results have been obtained also in experiments investigating tumor development around kidneys (Barclay et al., 2005). Additionally, studies within developmental biology show that the biomechanical context of the stroma can play essential roles in directing the development of the mammary gland and also influence the response to oncogenic mutations (Nelson \& Bissell, 2006). 
Final draft of a chapter to be published in D. S. Brooks, J. DiFrisco \& W. C. Wimsatt (Eds.), Biological Levels: Composition, Scale and Evolution in Complex Systems. MIT Press.

The ability of cancer cells to adapt or reprogram their expression profiles in response to their environment suggests that influences from the microenvironment in some cases can overrule genetic "instructions" and direct cell fate. The following sections examine how this has important practical as well as philosophical implications.

\section{Beyond the level of single cells: The Tumor Microenvironment}

In a widely cited Nature review, Bissell and Hines (2011) ask "Why don't we get more cancer?". The question is spurred by the observation that there are far fewer incidents of cancer than we should expect from the number of harmful mutations identified in cancer genomics. This has important implications for understanding the problem of overdiagnosis in cancer, i.e., for making sense of why some genetic or cellular abnormalities do not develop into symptomatically manifest disease (Green \& Vogt, 2016). A perspective beyond genetic is therefore needed to better understand the context of cancer development, including how higher-scale physical cues influence hierarchical control of cell proliferation.

Novel technologies to experimentally measure and manipulate physical cues have increased the ability of researchers to identify the effects of tissue stiffness. For instance, the development of 3D laminin rich gels to simulate the TME has allowed for systematic studies of the plasticity of cell populations that enable tumor cell reversion, depending on microenvironmental signals and tissue architecture (Nelson and Bissell 2005; 2006). A highly cited study by Paszek and colleagues (2005) illustrates this approach. The group used an electromechanical indentor to measure the relations between matrix stiffness and tumor development among colonies of mammary epithelial cells (MECs). The first step of the analysis compared normal breast tissues to malignant tissues in transgenic mice. The analysis showed that an average tumor, and stroma attached to tumors, are much stiffer on average (4049 $\mathrm{Pa} \pm$ 938) compared to a normal mammary gland $(167 \mathrm{~Pa} \pm 31)$.

To test whether stiff stroma was induced by genetic lesions, or whether the influence could go also in the opposite direction, Paszek et al. cultured normal MECs on gels with varying rigidity. They found that increased matrix stiffness significantly perturbed the tissue architecture (Figure 1). 
Final draft of a chapter to be published in D. S. Brooks, J. DiFrisco \& W. C. Wimsatt (Eds.), Biological Levels: Composition, Scale and Evolution in Complex Systems. MIT Press.

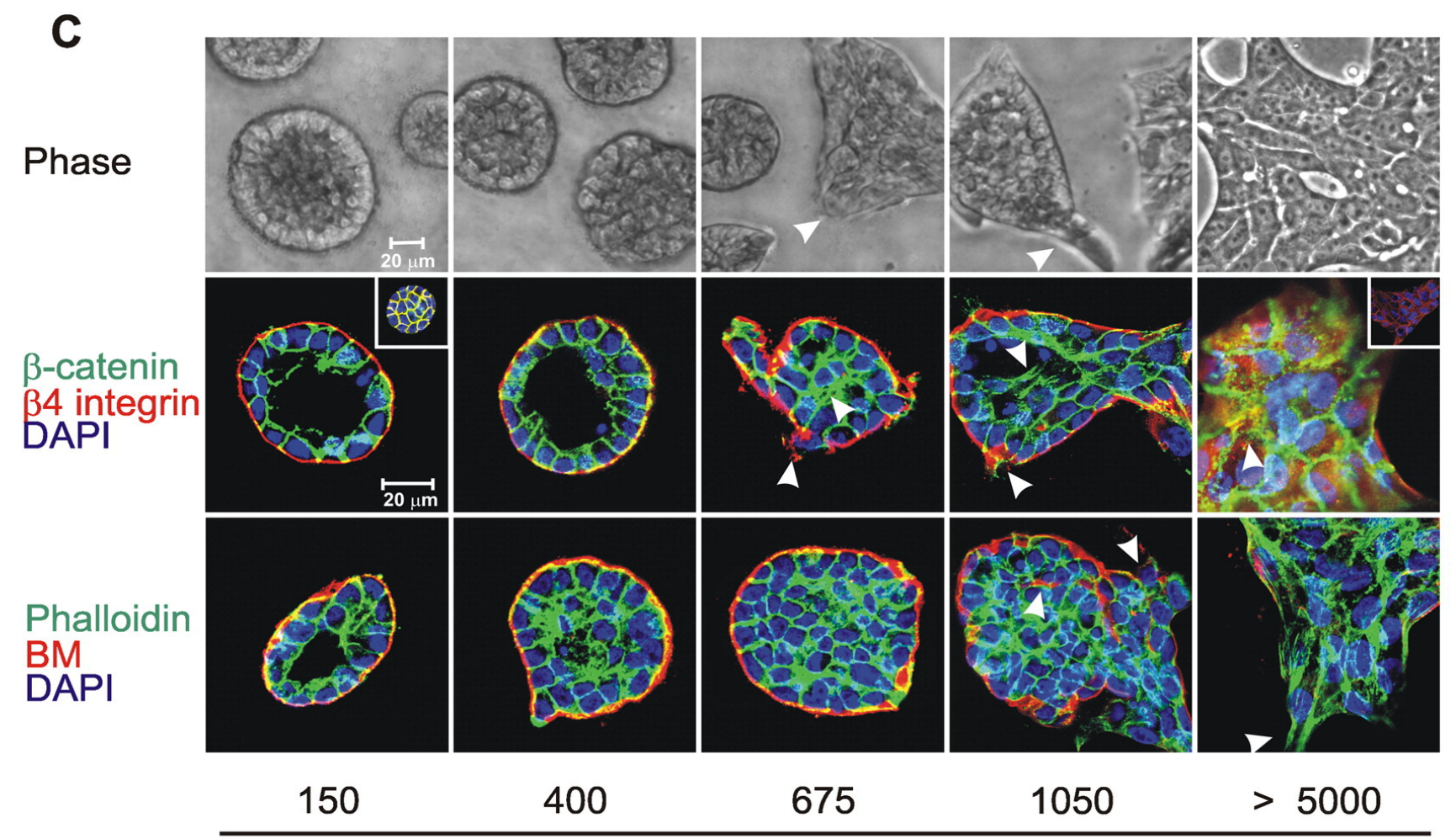

Elastic Modulus (Pa)

Figure 1. Phase contrast microscopy (top row) and confocal IF images of mammary epithelium cell cultures on gels of varying stiffness, represented as increasing values for elastic modulus (a measure of how much a material will elastically deform when subjected to stress). The images show the colony morphology after 20 days. Nuclei were stained with DAPI (blue coloring). The two bottom rows show cultures grown on collagen gels and basement membrane (BM) gels, respectively, using different staining techniques. The arrows highlight how increasing matrix stiffness (left to right) leads to destabilization of adherence junctions (green staining for $\beta$-catenin) and adhesion of integrins (red staining). Figure reprinted from (Paszek et al., 2005), with permission from Elsevier. A color version of the figure can be found in the online version of this chapter or in (Paszek et al. 2005, Figure 2C).

The experiment by Paszek et al. (2005) suggested that increased matrix stiffness destabilizes adhesion of cell junctions and integrins. Integrins are transmembrane ECM receptors that function as forcesensitive mechano-transducers by triggering cellular pathways. Accordingly, the group hypothesized that integrin alteration could lead to altered activity of proteins involved in growth-related molecular pathways. The study confirmed that cells with the malignant phenotype have higher activities of the Gprotein Rho and associated kinases (ROCK and MLC in Figure 2). 
Final draft of a chapter to be published in D. S. Brooks, J. DiFrisco \& W. C. Wimsatt (Eds.), Biological Levels: Composition, Scale and Evolution in Complex Systems. MIT Press.

Figure 2 highlights how integrin activation can trigger other molecular pathways stimulating cell proliferation, generating feedback that results in further stiffening of the matrix. The distinction between a "soluble" and "solid-state" signaling pathway emphasizes how studies of biochemical signaling pathways and of physical properties of the matrix, respectively, are considered equally important (Huang \& Ingber, 2005).

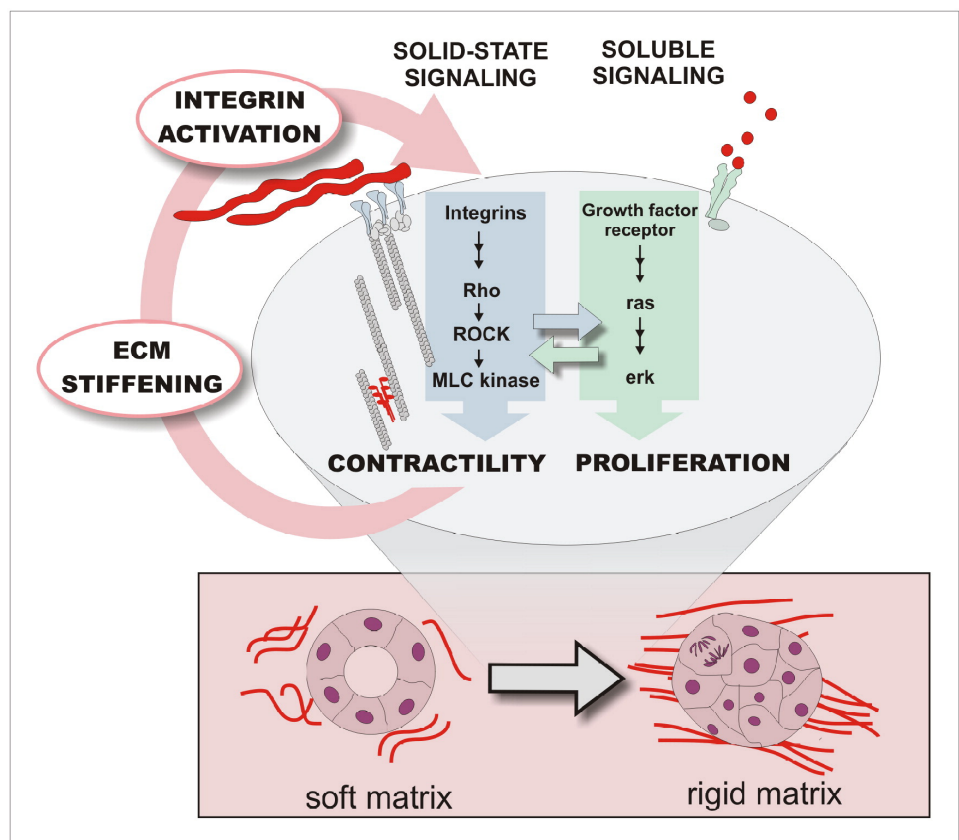

Figure 2. A mechanical autocrine loop that illustrates how ECM stiffening can lead to cancer development through a cascade of processes that further increases matrix rigidity. Reprinted from (Huang \& Ingber, 2005) with permission from Elsevier.

The recombination experiments discussed in Section 3 showed that transplantation of malignant cells to normal tissues can normalize cell-growth, and vice versa. Similarly, Paszek et al. (2005) demonstrated that the malignant phenotype can be strengthened or reverted, depending on the stiffness of the substrate. Approaches drawing the framework of condensed matter physics have more recently revealed that increased matrix stiffness correlates strongly with the development of breast cancer (Boyd et al., 2007), and that fluid and solid stresses influence the invasive potential of tumor cells (Rankin \& Frankel, 2017; West et al., 2017) as well as treatment response (Stylianopoulus 2017). Taken together, the results suggest an interpretation of the TME as a double-edged sword that can either protect from or promote cancer, depending its physical properties (Bissell \& Hines, 2011). ${ }^{5}$

\footnotetext{
${ }^{5}$ For other studies appealing to factors beyond genetic instability, see (Baker, 2013; Bizzarri \& Cucina, 2014; Egeblad et al., 2010; Laplane et al. 2018; Soto \& Sonnenschein, 2011).
} 
Final draft of a chapter to be published in D. S. Brooks, J. DiFrisco \& W. C. Wimsatt (Eds.), Biological Levels:

Composition, Scale and Evolution in Complex Systems. MIT Press.

It has recently been suggested that the physical properties of the TME offer new potential targets for cancer treatments (Bissell \& Hines, 2011; Rankin \& Frankel, 2016; Hirata \& Sahai, 2017;

Stylianopoulus, 2017). Following an initiative by the US National Cancer Institute in the late 1980s, antitumor drug screening is often carried out in 2D cultures. Yet, as highlighted in Section 3, the fate of cancer cells in response to carcinogenic or anti-carcinogenic influences may differ, depending on whether the cells are cultured in a petri dish or within the environmental context of the host organism. An important clinical implication of the experimental limitations of 2D cultures is that these cannot account for the development of drug resistance, which is very common in in vivo tumors. Limitations of $2 \mathrm{D}$ cultures has been suggested as part of the translational challenge that only 1-2 per 10.000 candidate drugs from antitumor drug screening currently make it further than Phase III studies (Feng et al., 2013).

In contrast, 3D cultures that can better account for the influence of biomechanical constraints on drug response (Nelson \& Bissell, 2006; Soto et al., 2008). For instance, the response of breast cancer cell lines to two antitumor drugs, cisplatin and taxol, has been demonstrated to depend on substrate stiffness (Feng et al., 2013). Recently, 3D hyaluronan gels have been used to mimic the tumor ECM in cell invasion studies. This line of research is inspired by the discovery that the physical properties of stromal components of cancer-resistant naked mole rats differ compared to humans (reviewed in Rankin \& Frankel, 2016). One of the important components of the microenvironment is hyaluronan, a glycosaminoglycan that can form gel like structures influencing the density and fluid pressure of the ECM. Chemical modifications of hyaluronan could potentially allow for interventions on the physical properties of the ECM and subsequently affect cell proliferation and cell invasion. Elevated solid stress has also been shown to create a fluid flux from tumors to surrounding tissues, and stress-alleviating stragies have been proposed as a way to improve treatment response (Stylianopoulus, 2017)

Compared to 2D cultures, 3D cultures are more demanding in terms of experimental resources and have other limitations (see Section 6). Yet, as argued in the following, they uncover aspects of cancer that may be interpreted as a form of downward causation. 
Final draft of a chapter to be published in D. S. Brooks, J. DiFrisco \& W. C. Wimsatt (Eds.), Biological Levels: Composition, Scale and Evolution in Complex Systems. MIT Press.

\section{Downward causation}

How can we make sense of the role of tissue-scale biomechanical factors in the context of cancer? I suggest that the role of tissue-scale biomechanics can be interpreted as an instance of downward causation. Importantly, however, the account defended is different from the "strong" account criticized by Kim (1998) and others, which entails synchronic efficient causation across levels and ontological autonomy of higher-level wholes of lower-level parts. Instead, I consider downward causation as a relation between system variables operating at different scales. This suggestion is in line with Woodward's account (this volume) that highlights effects on lower-level variables through manipulation of high level variables as the criteria for downward causation (see also Ellis, 2012). The experiments presented in Sections 3 and 4 are examples of interventions on higher-scale factors (tissue stiffness) that influence the behavior of lower-scale variables (gene expression and cellular behaviors) and thus satisfy this criterion.

Top-down influences, in my view, are best understood as constraining relations that "select and delimit various types of the system's possible developments" (Emmeche et al., 2000, p. 25; see also Brooks \& Eronen, 2018). This account is also inspired by systems biologist Denis Noble's suggestion that downward causation can be interpreted mathematically "as the influences of initial and boundary conditions on the solutions of the differential questions used to represent the lower level processes" (Noble 2012, p. 55; see also Ellis, 2012). The account is developed in more detail in a separate paper on downward causation in the context of multi-scale cardiac modeling (Green, 2018). ${ }^{6}$ Here, I primarily wish to defend the explanatory importance of higher-level constraints.

By constraints, I understand features that delimit the degree of freedom for a given (Hooker, 2013; Umerez \& Mossio, 2013). Constraints not only limit possibilities through restraints on the possible system states but also enable certain states that would be impossible to reach for the unconstrained system. For instance, Noble highlights that the oscillating dynamics of the heart rhythm cannot be

\footnotetext{
${ }^{6}$ As discussed by Mark Eronen at the meeting at the KLI, it can be debated whether one should insist on the term downward causation on this basis. I have no strong stance on this, but I hope to show that interpreting downward causation through constraining relations does not weaken the causal and explanatory power of top-down effects (Ellis 2012).
} 
Final draft of a chapter to be published in D. S. Brooks, J. DiFrisco \& W. C. Wimsatt (Eds.), Biological Levels: Composition, Scale and Evolution in Complex Systems. MIT Press.

generated (or explained) without the boundary of cell structure, which creates a concentration gradient (cell voltage or cell potential) across the cell membrane. He argues that "without the downward causation from the cell potential, there is no rhythm" (Noble, 2012, p. 58).

Physical constraints are often mathematically represented as boundary conditions that impose limits on the domain of the model. These typically cannot be defined at the lowest explanatory scale. For instance, in Noble's example of the heart rhythm, the cell potential is a variable that cannot be understood or measured at the genetic level. It is a parameter that has to be measured at a higher scale (e.g., through microelectrode measurements of intact or coupled cells). Without imposing boundary conditions on the solutions of lower-scale models (in this context ordinary differential equations), the lower-scale models cannot be solved. Medium downward causation thus reveals important limitations to a reductionist perspective - by demonstrating the explanatory limitations of microscale details even for models at the lowest scale.

The requirement of higher scale parameters is not unique to biology. Macro-scale parameters are also indispensable when modeling physical behaviors, such as steady state heat conduction in a rod (McGivern, 2008) or drop-formation (Batterman, 2006; for other examples see Batterman, 2012; 2018). In the context of multi-scale cancer models, an important macro-scale parameter is matrix stiffness, defined as Young's modulus (also called the elastic modulus), which outlines how much a material will deform in response to stress as a property evenly distributed over a given material's surface (Deisboeck \& Stamatakos, 2011). The stiffness of a material, whether the material is steel or biological tissues, is a macro-scale parameter that identifies elastic properties by treating structure as a larger continuum of matter (Green \& Batterman, 2017). In our examples here, matrix stiffness is inherently a tissue-scale parameter because it depicts the physical forces acting on the integrated effects of cell populations that are constrained by certain geometrical structures (Davidson, von Dassow, \& Zhou, 2009). Other examples of macroscale parameters used in continuum models of tumor dynamics are forces of cell-cell adhesion, migration velocity of cells, ECM fiber thickness and length variation (Deisboeck \& Stamatakos, 2011; West et al., 2017). Whenever the output of models or experiments targeting lower scales (e.g., gene regulatory networks) is influenced by changes in high level variables such as tissue stiffness, I would argue that we have an instance of "downward" regulation of cell behavior. 
Final draft of a chapter to be published in D. S. Brooks, J. DiFrisco \& W. C. Wimsatt (Eds.), Biological Levels:

Composition, Scale and Evolution in Complex Systems. MIT Press.

The need for multiple approaches not only results from the complexity of biological systems but also from what, in the context of physics, has been called the "tyranny of scales" (Oden, 2006). The problem refers to the scale-dependency of physical properties as well as the conceptual frameworks we understand these through. Batterman (2012) highlights that when modeling physical systems, such as a steel beam, it is not possible to describe all relevant factors in one overarching mathematical framework. Steel exhibits different physical properties at the atomic, intermediate, and macroscales, and capturing all aspects requires that different models are employed. For instance, continuum models describing elastic macroscale properties of steel treat the material as a continuum without discrete structures. But an examination of the structure of steel at a mesoscale reveals grain boundaries, cracks etc. Because physical behaviors and our concepts used to describe these are "multi-valued" across scales (Wilson, 2012), researchers are inevitably forced to combine different mathematical frameworks to account for structures and processes across scales.

The situation is analogous in the context of cancer. Young's modulus is a continuum parameter that treats materials as if they were continuous rather than made up discrete parts. Obviously, biological systems consist of very different parts with different properties, and such crude idealizations may seem misguided. But a striking insight is that coarse-grained models often work well, despite of (and often because of) such simplifications (Batterman, 2018). Continuum models necessarily abstract from lower-scale details to identify parameters operating at higher scales. Importantly, and as further defended below, matrix stiffness cannot be understood simply as the aggregated effects of molecular actions (see also Batterman \& Green, forthcoming). Relative autonomy of scales or levels is thus one of the key factors in support of downward causation. In the following, I consider and reply to potential objections to this point.

\section{Potential objections and replies}

A reductionist could object that since a tumor consists of nothing but molecular constituents, a more fundamental model targeting lower scales should in principle be sufficient. Such "in principle arguments" are, however, not particularly convincing or interesting if they are not accompanied by a clarification of how one would conduct such a reduction in practice (see also Batterman, 2018). 
Final draft of a chapter to be published in D. S. Brooks, J. DiFrisco \& W. C. Wimsatt (Eds.), Biological Levels:

Composition, Scale and Evolution in Complex Systems. MIT Press.

Particulalry, the reductionist has to clarify how upper-level variables are supposed to be reduced to lower-level variables.

One problem for the reduction of upper-level variables is multiple realizability, i.e., that a given upperlevel variable has a number of different lower-level "realizers" (Woodward, this issue). An important consequence of multiple realizability is that much of the variation at a micro level does not matter for the study of macroscale properties. Woodward refers to such cases as instances of conditional independence. In the context of cancer research, the dynamic state of given cancer phenotype may be realized by multiple molecular states, and biochemical details do not always matter for prediction of the tissue-scale effects (West et al., 2017). The reductionist thus has to explain how it is possible to have this sort of explanatory autonomy (Batterman, 2018). ${ }^{7}$

Another important problem for the reductionist is that many higher-level variables are irreducible in the sense that they cannot be measured or conceptualized at lower scales (Ellis, 2012). I began the paper with a quote from Smithers (1962) arguing that cancer is no more a disease of cells than a traffic jam is a disease of cars. In both contexts, studying the constituents of the individual components, cars or cells, would not allow us to explain why the problem of cancer or traffic jams arise. Both are macroscale phenomena that we make sense of by looking at higher-level relations between the constituents, involving structural constraints such as roads and tissue boundaries. The examples are analogous to how the rules of a competitive sports game cannot be derived from detailed studies of the behavior of individuals (Sawyer, 2002), or how properties such as the temperature or pressure of a gas cannot be ascribed to individual gas molecules (Christiansen, 2000). The reductionist therefore has to clarify how we can make sense of such macroscale phenomena without reference to higher-level concepts and parameters.

\footnotetext{
${ }^{7}$ As highlighted by Woodward, multiple realizability need not make higher-level explanations inferior, since explanatory relevance is dependent on epistemic aims that define the criteria for conditional relevance or irrelevance. Moreover, as illustrated by Figure 2, one often needs to account for multiple causal factors operating in what Woodward calls causal cycles (i.e., where influences go in both directions). But it does present a challenge for the view that features at the lowest scale are sufficient.
} 
Final draft of a chapter to be published in D. S. Brooks, J. DiFrisco \& W. C. Wimsatt (Eds.), Biological Levels: Composition, Scale and Evolution in Complex Systems. MIT Press.

One strategy to respond to such examples in support of a reductionist view is to say that although constraints from higher-scale structures play some causally relevant role, it does not automatically grant top-down effects an explanatory role in science. A perspective giving explanatory priority to molecular mechanisms might, for instance, interpret the result of the experiments by Paszek et al. (2005) differently. It might be argued that the physical forces of tissue structures are just background conditions for the most important difference-makers, namely the effects of increased Rho-activity on molecular pathways of cell cycle progression. ${ }^{8}$

Privileging genetic causation is motivated by an epistemic ideal in biology that emphasize cellautonomous or "instructive" molecular information. In cancer research this ideal is reflected in the prioritization of "intrinsic" factors of carcinogenesis. Whereas this ideal has its merits for answering some explanatory questions, it may be counterproductive for addressing clinically relevant aspects of cancer. Methodological reduction, i.e., strategies to ignore or simplify some aspects in order to focus on others, is a necessary requirement for any scientific analysis of complex systems (Wimsatt, 2007). But if one is unaware of the existence of blind spots created through specific theoretical and experimental choices, there is a risk of committing what Wimsatt at the KLI-meeting called functional localization fallacies. The term refers the common bias of downgrading factors that - as a practical necessity - are left out of scientific analysis. The rejection of the explanatory role of tissue biomechanics may partly result from a functional localization fallacy.

Biomechanical factors are often ignored or held fixed as a practical requirement for experimental intervention on genetic factors (Robert, 2004). Hence, the results of such experiments cannot be informative of the relative importance or (ir)relevance of biomechanical factors as these are excluded from the outset of the analysis. As mentioned, genetic difference-making is often studied through 2D cultures of cell populations with controlled and modified gene expression, e.g., via gene knock-out experiments (Vaux, 2011). Such studies, together with more recent tumor sequencing projects, have no doubt shed light on important genetic markers and pathways involved in carcinogenesis. But as it is now well established that epithelial cells behave differently if placed in a dish coated with basement

\footnotetext{
${ }^{8}$ Such an argument has been put forward in philosophical discussions about reductionism, e.g., by pointing out that macroscale features are merely background factors in molecular developmental biology (Rosenberg, 1997).
} 
Final draft of a chapter to be published in D. S. Brooks, J. DiFrisco \& W. C. Wimsatt (Eds.), Biological Levels:

Composition, Scale and Evolution in Complex Systems. MIT Press.

membrane proteins or in cultures mimicking the TME rather than cultured on a plain plastic dish (Plutynski 2018a, p. 41; 2018b), reference to the success of the gene-centric line of research cannot support the rejection of an explanatory role of physical features of the TME. Traditional gene-centric experimental designs are thus inherently limited when it comes to accounting for the role of macroscale physical factors in the natural environment of the cells (Soto \& Sonnenschein, 2011; see Brigandt \& Love, 2017 for a more general discussion of reductionism in biology).

As mentioned in Section 4, alternative attempts involve building higher-dimensional models, such as a 3D model of the mammary gland and hyaluronan gels to mimic the TME (Nelson \& Bissell, 2005; Rankin \& Frankel, 2016). Moving to the tissue scale, however, typically requires that many molecular details are ignored, and the experiments are more laborious and difficult to automate. They would also become practically intractable for studying the effects, and combined effects, of hundreds or even thousands of genetic difference makers. The key point is here that different experimental strategies foreground specific (and often complementary) aspects of a complex system, here ranging from the influence of biomechanical cues on cell differentiation to genetic difference making.

Cancer is characterized by what Wimsatt (1972) calls interactional complexity. Although different experimental and theoretical approaches are compatible, different ways of investigating the system often require distinct ways of demarcating and decomposing the phenomenon (Plutynski, 2018a). As a result, the different perspectives are not straightforwardly integrated. When Huang and Ingber distinguish between two forms of signaling (Figure 2), it is because they wish to highlight that the physics perspective is not reducible to a biological perspective, or vice versa. Whereas some would see the biomechanical constraints merely as background conditions, they emphasize that Paszek's interventions demonstrate the causal relevance of a "physical cue devoid of chemical specificity" (Huang \& Ingber, 2005, p. 176).

The studies examined put forward the possibility that the architecture and mechanical properties of the tissues can be dominant over genotype, because malignant phenotypes can sometimes be reverted through interventions that alter microenvironmental factors (Nelson and Bissel, 2006; Paszek et al., 2005; Bissell \& Hines, 2011). This suggests that physical factors of the TME cannot generally be 
Final draft of a chapter to be published in D. S. Brooks, J. DiFrisco \& W. C. Wimsatt (Eds.), Biological Levels:

Composition, Scale and Evolution in Complex Systems. MIT Press.

considered merely as background conditions of limited explanatory and predictive value. A similar argument has been defended in the contexts of multi-scale modeling in developmental biology (Green $\&$ Batterman, 2017) and cardiac models systems biology (Green, 2018).

Multi-scale models combine different models targeting different scales. These models are at the same time "explanatorily independent" and "epistemologically interdependent" (Potochnik, 2009). They are explanatorily independent in the sense that they legitimately ignore some aspects to address questions or properties at specific scales or levels. For instance, macroscale models are autonomous in the sense that they are often not improved by incorporation of all possible molecular details (Batterman, 2018). Similarly, microscale models typically black box many higher-scale features as these (autonomously) describe a process from a micro-level perspective.

At the same time, models targeting different scales in a multi-scale modeling approach can be said to be "epistemologically interdependent", because they often rely on sources of information provided by other sources or models (Potochnik, 2009). A model at a characteristic scale often needs inputs or boundary conditions defined by models of a different spatial (or temporal) scale (Lesne, 2013). In cancer research, multi-scale models must integrate continuum and discrete models to capture both large-scale volumetric tumor growth dynamics and behaviors of individual and heterogeneous cells (Deisboeck \& Stamatakos, 2011). Because each framework presents a simplified picture of a system's dynamics, their applicability may depend upon factors that the models themselves do not represent or capture. Lower-scale models often require macroscale inputs and vice versa, and each perspective foregrounds some aspects while ignoring or idealizing others.

As a consequence of the tyranny of scales problem and interactional complexity, it seems futile to look for the origin of cancer or to debate what constitutes a "right level" of analysis. Different perspectives, addressing different scales using methods from different disciplines, can answer different types of explanatory questions. Each model addressing specific aspects at a given spatial (or temporal) scale will black box other questions or aspects at different scales. But if one is not aware of such "black boxing procedures", it may result in theoretical biases. It could be argued that the current privileging of genetic factors is an example of such a bias, and that a more balanced view is needed. 
Final draft of a chapter to be published in D. S. Brooks, J. DiFrisco \& W. C. Wimsatt (Eds.), Biological Levels:

Composition, Scale and Evolution in Complex Systems. MIT Press.

\section{Towards a multi-scale perspective on cancer?}

Debates on cancer are often framed as a choice between alternatives, i.e., between views of cancer as a genetic or tissue-based disease. However, the concept of the TME can encompass several types of influences on cell differentiation, spanning from biochemical influences from the surrounding cells to mechanical and field forces (stiffness and surface tension) and topological geometry of the tissue (Bizzarri \& Cucina, 2014; Laplane et al., 2018). Thus, an understanding of cancer across scales need not privilege any one level of causation (Bertolaso, 2011; Malaterre, 2011).

My aim in this chapter has not been to reject the merits of reductionist approaches. Not all explanatory questions require higher-level analysis, and gene-centric approaches have brought about many important insights to the development and treatment of cancer. Yet, the predictive capacities for clinical applications often depend on the willingness to embrace complexity. In other words, interactional complexity also has practical implications for how many theoretical perspectives are required to predict or explain the behavior of the system (Wimsatt, 1972; Plutynski, 2018a). Accepting a pluralistic stance should therefore not bar us from questioning the scope or sufficiency of the dominant gene-centric approach.

A consequence of specialization and complexity in science is often that narrow explanatory questions are pursued in different settings, with limited attempts to integrate the various research results. Thousands of papers are published each year on cancer, many with a focus on how specific genes or proteins contribute to a specific cancer-related pathway. But it has proven tremendously challenging to synthesize insights from these studies into clinically relevant applications (Lazebnik, 2002; 2010; Prasad et al., 2016). Accordingly, the debate on the relevant theoretical perspectives on cancer is not only philosophically interesting but is also of practical importance to the design of experiments and prospects of funding strategies. As stated by one of key pioneers of the research program focused on the TME:

One of the first steps in our attempts to comprehend the big picture of tumor progression is to realize that single molecules or single signaling pathways are just solitary components of an 
Final draft of a chapter to be published in D. S. Brooks, J. DiFrisco \& W. C. Wimsatt (Eds.), Biological Levels:

Composition, Scale and Evolution in Complex Systems. MIT Press.

immense network. This realization should lead to the abandonment of reductionism (which, I am afraid, is a difficult mission under the present culture of conducting science and its funding), and to the employment of approaches used in Systems Biology" (Witz, 2009, S13)

Similarly, Bizarri and Cucina (2014) highlight that the pharmaceutical industry, focused on the development of drugs targeting specific molecular components, is strengthening the bottom-up reductionist approach at the expense of other potentially useful approaches. Explanatory priorities regarding specific levels of analysis are therefore also related to the problem of opportunity cost, i.e. to the problem of how to prioritize investments in different solution strategies. An important job for philosophy of science could thus be to examine the merits of assumptions underlying such prioritizations of specific levels of analysis. Another could be to examine the potial or relevance of integrating different perspectives. Given the complexity of cancer, it cannot be assumed that the development of multi-scale models will easily solve the translation problem.

Multi-scale modeling is particularly challenging in biology, compared to modeling of many physical systems, because there are complex non-linear feedback relations that crosscut spatial and temporal scales. Moreover, biological systems have active boundaries that change over time as an organism responds to environmental stimuli, develops, and ages. This means that the causal effects of any type of difference-maker may be highly context-sensitive. In the context of cancer, a given genetic instability may for instance have very different effects depending on the age stage of a person as ageing involves changes in stiffness and elasticity of tissues (Bissell \& Hines, 2011). Cancer is a "fast moving target" that develops from a variety of causes, in a variety of ways, and at various levels (Bertolaso, 2011), and is hence especially challenging to model.

\section{Summary and concluding remarks}

Theoretical assumptions concerning the causal nature of cancer can influence choices of experimental designs to study cancer - and vice versa. Functional localization of specific features of complex systems necessarily foreground some aspects while backgrounding others. In cancer research, 2D 
Final draft of a chapter to be published in D. S. Brooks, J. DiFrisco \& W. C. Wimsatt (Eds.), Biological Levels:

Composition, Scale and Evolution in Complex Systems. MIT Press.

cultures are efficient tools for studying effects of gene knockout and other molecular manipulations on cell proliferation. Yet, this approach is fundamentally limited in revealing environmental influences on tumor progression and drug response. Recombination experiments in vivo and studies of $3 \mathrm{D}$ cultures in vitro a have led to important theoretical insights as well as to suggestions of new types of cancer treatment. Each framework is, however, only partial. Although they can be considered explanatorily independent, i.e., merited by particular questions of analysis, they may be considered epistemically interdependent in the sense that improving predictions of one model may rely on inputs from other models or other experimental sources.

This chapter has focused on the role of biomechanical features of the TME for cancer progression. I believe that the cases presented provide an interesting set of examples for philosophical discussions on the explanatory role and autonomy of macroscale features, as well as for discussions on the relative importance of biological and physical science approaches to cancer (see also Love et al., 2017). Contrary to the so-called layer-cake model of science, physical science approaches do not always play a theory-reducing role. On the contrary, in this context physical science approaches highlight the importance of macroscale properties. I have argued that the influence of tissue-scale biomechanical constraints on gene expression and direction of cell fate can be interpreted as a form of downward causation. Specifically, I have defended an account of downward causation that can be mathematically interpreted as the influences of boundary conditions on the solutions of lower-scale models.

As a consequence of the interactional complexity of cancer, the results of the many efforts may not make up a neatly integrated causal mosaic of various factors (Love, 2017). The resulting picture may be more akin to a fragmented cubist painting where some aspects of the system are yet to become fully visible for scientific analysis. ${ }^{9}$ While this may be seen as a disappointing conclusion, it does not mean that improvements cannot be reached. Multi-scale approaches to cancer call for caution against the stubbornly persistent idea that genetic mutations constitute the most relevant or most reliable feature of

\footnotetext{
${ }^{9}$ I would like to thank Leen de Vreese for suggesting the analogy of a cubist painting, inspired by the work of Caterina Marchionni, University of Helsinki.
} 
Final draft of a chapter to be published in D. S. Brooks, J. DiFrisco \& W. C. Wimsatt (Eds.), Biological Levels: Composition, Scale and Evolution in Complex Systems. MIT Press.

cancer. A perspective from higher-scale tissue biomechanics allows for other visible patterns to materialize, which is not only theoretically intriguing but also of clinical relevance.

\section{References}

Baker, S. G. (2012). Paradoxes in carcinogenesis should spur new avenues of research: an historical perspective. Disruptive science and technology, l(2), 100-107.

Baker, S. G. (2013). Paradox-driven cancer research. Disruptive science and technology, 1(3), 143-148.

Barclay, W. W., Woodruff, R. D., Hall, M. C., \& Cramer, S. D. (2005). A system for studying epithelial-stromal interactions reveals distinct inductive abilities of stromal cells from benign prostatic hyperplasia and prostate cancer. Endocrinology, 146(1), 13-18.

Batterman, R. W. (2006). Hydrodynamics versus molecular dynamics: Intertheory relations in condensed matter physics. Philosophy of Science, 73, 888-904.

Batterman, R. W. (2012). The tyranny of scales. In R. W. Batterman (Ed.), Oxford Handbook of Philosophy of Physics (pp. 255-286). Oxford: Oxford University Press.

Batterman, R. W. (2018). Autonomy of theories: An explanatory problem. NOUSS, 52: 4, 858-873.

Batterman, R. W. \& Green, S. (forthcoming). Steel and Bone: Mesoscale modeling and middle-out strategies in physics and biology. Synthese.

Bedessem, B., \& Ruphy, S. (2015). SMT or TOFT? How the two main theories of carcinogenesis are made (artificially) incompatible. Acta Biotheoretica, 63(3), 257-267.

Bedessem, B., \& Ruphy, S. (2017). SMT and TOFT integrable after all: A reply to Bizzarri and Cucina. Acta Biotheoretica, 65(1), 81-85.

Bertolaso, M. (2011). Hierarchies and causal relationships in interpretative models of the Neoplastic Process. History and Philosophy of the Life Sciences, 33, 515-536.

Bissell, M. J., \& Hines, W. C. (2011). Why don't we get more cancer? A proposed role of the microenvironment in restraining cancer progression. Nature medicine, 17(3), 320-329.

Bizzarri, M., \& Cucina, A. (2014). Tumor and the microenvironment: a chance to reframe the paradigm of carcinogenesis? BioMed research international, 2014.

Bizzarri, M., \& Cucina, A. (2016). SMT and TOFT: Why and how they are opposite and incompatible paradigms. Acta Biotheor, 64(3), 221-239.

Blanchard, A., \& Strand, R. (Eds.). (2017). Cancer Biomarkers: Ethics, Economics and Society. Kokstad: Megaloceros.

Boyd, N. F., Guo, H., Martin, L. J., Sun, L., Stone, J., Fishell, E., . . Yaffe, M. J. (2007). Mammographic density and the risk and detection of breast cancer. New England Journal of Medicine, 356(3), 227-236.

Brigandt, I., \& Love, A. C. (2017). Reductionism in Biology. Stanford Encyclopedia of Philosophy, https://plato.stanford.edu/entries/reduction-biology/.

Brooks, D., \& Eronen, M. (2018). Levels of organization in biology. Stanford Encyclopedia of Philosophy, https://plato.stanford.edu/entries/levels-org-biology/.

Campbell, D. T. (1974). 'Downward causation' in hierarchically organised biological systems. In F. Ayala \& T. Dobzhansky (Eds.), Studies in the Philosophy of Biology (pp. 179-186): University of California Press. 
Final draft of a chapter to be published in D. S. Brooks, J. DiFrisco \& W. C. Wimsatt (Eds.), Biological Levels:

Composition, Scale and Evolution in Complex Systems. MIT Press.

Christiansen, P. V. (2000). Macro and micro-levels in physics. In P. B. Andersen, C. Emmeche, N. O. Finnemann, \& P. V. Christiansen (Eds.), Downward Causation. Minds, Bodies and Matter (pp. 51-62). Aarhus: Aarhus University Press.

Davidson, L., von Dassow, M., \& Zhou, J. (2009). Multi-scale mechanics from molecules to morphogenesis. The international journal of biochemistry \& cell biology, 41(11), 2147-2162.

Deisboeck, T., \& Stamatakos, G. (2011, eds.). Multiscale cancer modeling. Boca Raton, FL. CRC Press.

DiFrisco, J. (2017). Time scales and levels of organization. Erkenntnis, 82(4), 795-818.

Dolberg, D. S., \& Bissell, M. J. (1984). Inability of Rous sarcoma virus to cause sarcomas in the avian embryo. Nature, 309(5968), 552-556.

Egeblad, M., Nakasone, E. S., \& Werb, Z. (2010). Tumors as organs: complex tissues that interface with the entire organism. Developmental cell, 18(6), 884-901.

Ellis, G. F. R. (2012). Top-down causation and emergence: some comments on mechanisms Interface Focus, 2(1), 126-140.

Emmeche, C., Køppe, S. and Stjernfelt, F. (2000). Levels, emergence, and three versions of downward causation. In P. B. Andersen, C. Emmeche, N. O. Finnemann, \& P. V. Christiansen (Eds.), Downward Causation. Minds, Bodies and Matter (pp. 13-34). Aarhus: Aarhus University Press.

Feng, J, Tang, Y., Xu, Y., Sun, Q., Liao, F. \& Han, D. (2013). Substrate stiffness influences the outcome of antitumor drug screening in vitro. Clinical Hemorheology and Microcirculation, 55, 121-131.

Folkman, J. (1971). Tumor angiogenesis: therapeutic implications. New England Journal of Medicine, 285(21), 1182-1186.

Green, S. (2018). Scale-dependency and downward causation in biology. Philosophy of Science, 85 (Dec 2018).

Green, S., \& Batterman, R. (2017). Biology meets physics: Reductionism and multi-scale modeling of morphogenesis. Studies in History and Philosophy of the Biological and Biomedical Sciences, 61, 20-34.

Green, S., \& Vogt, H. (2016). Personalizing medicine: Disease prevention in silico and in socio. Humana.Mente Journal of Philosophical Studies, 9(30), 105-145.

Greenman, C., Stephens, P., Smith, R., Dalgliesh, G. L., Hunter, C., Bignell, G., . . Stratton, M. R. (2007). Patterns of somatic mutation in human cancer genomes. Nature, 446(7132), 153-158.

Hanahan, D., \& Weinberg, R. A. (2011). Hallmarks of cancer: the next generation. Cell, 144(5), 646674.

Hirata, E., \& Sahai, E. (2017). Tumor microenvironment and differential responses to therapy. Cold Spring Harbor perspectives in medicine, 7(7), a026781.

Hooker, C. (2013). On the import of constraints in complex dynamical systems. Foundations of Science, 18(4), 757-780.

Huang, S., \& Ingber, D. E. (2005). Cell tension, matrix mechanics, and cancer development. Cancer Cell, 8(3), 175-176.

Kaiser, M. (2015). Reductive Explanation in the Biological Sciences. Springer International Publishing Switzerland: Springer.

Kim, J. (1998). Mind in a Physical World. Cambridge, MA.: MIT Press.

Laplane, L., Duluc, D., Larmonier, N., Pradeu, T., \& Bikfalvi, A. (2018). The multiple layers of the tumor environment. Trends in Cancer, 4(1), 802-809. 
Final draft of a chapter to be published in D. S. Brooks, J. DiFrisco \& W. C. Wimsatt (Eds.), Biological Levels:

Composition, Scale and Evolution in Complex Systems. MIT Press.

Lazebnik, Y. (2002). Can a biologist fix a radio? - Or, what I learned while studying apoptosis. Cancer Cell, 2(3), 179-182.

Lazebnik, Y. (2010). What are the hallmarks of cancer? Nature Comment, 10, 232-233.

Lesne, A. (2013). Multiscale analysis of biological systems. Acta Biotheoretica, 61(1), 3-19.

Lochter, A., \& Bissell, M. J. (1995). Involvement of extracellular matrix constituents in breast cancer. Seminars in Cancer Biology, 3(6), 165-173.

Love, A. C. (2012). Hierarchy, causation and explanation: ubiquity, locality and pluralism. Interface Focus, 2(1), 115-125.

Love, A. C. (2017). Building integrated explanatory models of complex biological phenomena: From Mill's methods to a causal mosaic. In EPSA15 Selected Papers. European Studies in Philosophy of Science (Vol. 5, pp. 221-232). Cham: Springer.

Love, A. C. (this issue). Manipulating levels of organization. In D. S. Brooks, J. DiFrisco \& W. C. Wimsatt (Eds.), Biological Levels: Composition, Scale and Evolution in Complex Systems. MIT Press.

Love, A. C., Stewart, T. A., Wagner, G. P., \& Newman, S. A. (2017). Perspectives on integrating genetic and physical explanations of evolution and development: An introduction to the symposium. Integrative and Comparative Biology, 57(6), 1258-1268.

Maffini, M. V., Calabro, J. M., Soto, A. M., \& Sonnenschein, C. (2005). Stromal regulation of neoplastic development: age-dependent normalization of neoplastic mammary cells by mammary stroma. American Journal of Pathoogyl, 167(5), 1405-1410.

Maffini, M. V., Soto, A. M., Calabro, J. M., Ucci, A. A., \& Sonnenschein, C. (2004). The stroma as a crucial target in rat mammary gland carcinogenesis. Journal of Cell Science, 117(8), 14951502.

Malaterre, C. (2011). Making sense of downward causation in manipulationism: illustrations from cancer research. History and Philosophy of the Life Sciences, 33(4), 537-561.

McGivern, P. (2008). Levels of reality and scales of application. In B. Ellis, H. Sankey, \& A. Bird (Eds.), Properties, powers and structures: Issues in the metaphysics of realism (pp. 45-60). New York: Routledge.

Miklos, G. L. G. (2005). The human cancer genome project--one more misstep in the war on cancer. Nat Biotechnol, 23(5), 535-537. doi:10.1038/nbt0505-535

Montévil, M., \& Pocheville, A. (2017). The Hitchhiker's guide to the cancer galaxy: How two critics missed their destination. Organisms. Journal of Biological Sciences, 1(2), 37-46.

Mossio, M., Bich, L., \& Moreno, A. (2013). Emergence, closure and inter-level causation in biological systems. Erkenntnis, 78(2), 153-178.

Nelson, C. M., \& Bissell, M. J. (2005). Modeling dynamic reciprocity: engineering three-dimensional culture models of breast architecture, function, and neoplastic transformation. Seminars in Cancer Biology, 15(5), 342-352.

Nelson, C. M., \& Bissell, M. J. (2006). Of extracellular matrix, scaffolds, and signaling: tissue architecture regulates development, homeostasis, and cancer. Annual Review of Cell and Developmental Biology, 22, 287-309.

Noble, D. (2012). A theory of biological relativity: no privileged level of causation. Interface focus, 2(1), 55-64.

Oden, J. T. (Chair) (2006). Simulation based engineering science - An NSF Blue Ribbon Report. www.nsf.gov/pubs/reports/sbes_final_report.pdf. 
Final draft of a chapter to be published in D. S. Brooks, J. DiFrisco \& W. C. Wimsatt (Eds.), Biological Levels: Composition, Scale and Evolution in Complex Systems. MIT Press.

Paszek, M. J., Zahir, N., Johnson, K. R., Lakins, J. N., Rozenberg, G. I., Gefen, A., .. . Weaver, V. M (2005). Tensional homeostasis and the malignant phenotype. Cancer Cell, 8(3), 241-254.

Plimmer, H. G. (1903). The parasitic theory of cancer. British Medical Journal, 2(2241), 1511-1515.

Plutynski, A. (2018a). Explaining cancer. Finding order in disorder. New York, NY: Oxford University Press.

Plutynski, A. (2018b). The origins of "dynamic reciprocity": Mina Bissell's expansive picture of cancer causation. In Harman, O. \& Dietrich, M. (eds.). Dreamers, Visionaries, and Revolutionaries in the Life Sciences (pp. 96-109), University of Chicago Press.

Potochnik, A. (2009). Explanatory independence and epistemic interdependence: A case study of the optimality approach. British Society for the Philosophy of Science, 61(June 18), 213-233.

Potochnik, A., \& McGill, B. (2012). The limitations of hierarchical organization. Philosophy of Science, 79(1), 120-140.

Prasad, V., Fojo, T., \& Brada, M. (2016). Precision oncology: origins, optimism, and potential. The Lancet Oncology, 17(2), e81-e86.

Qiu, W., Hu, M., Sridhar, A., Opeskin, K., Fox, S., Shipitsin, M., . . Gorringe, K. L. (2008). No evidence of clonal somatic genetic alterations in cancer-associated fibroblasts from human breast and ovarian carcinomas. Nature Genetics, 40(5), 650.

Rankin, K. S., \& Frankel, D. (2016). Hyaluronan in cancer - from the naked mole rat to nanoparticle therapy. Soft Matter, 12(17), 3841-3848.

Robert, J. S. (2004). Embryology, Epigenesis, and Evolution: Taking Development Seriously. New York: Cambridge University Press.

Rosenberg, A. (1997). Reductionism redux: Computing the embryo. Biology and Philosophy, 12, 445 470.

Sawyer, K. R. (2002). Nonreductive individualism: Part I - Supervenience and wild disjunction. Philosophy of the Social Sciences, 32, 537-559.

Smithers, D. W. (1962). An attack on cytologism. Lancet, 1(7228), 493-499.

Sonnenschein, C., \& Soto, A. (1999). The Society of Cells: Cancer and Control of Cell Proliferation. New York: Springer-Verlag.

Sonnenschein, C., Soto, A. M., Rangarajan, A., \& Kulkarni, P. (2014). Competing views on cancer. Journal of Bioscience, 39(2), 281-302.

Soto, A., \& Sonnenschein, C. (2011). The Tissue Organization Field Theory of cancer: A testable replacement for the Somatic Mutation Theory. BioEssays, 5, 322-340.

Soto, A. M., Sonnenschein, C., \& Miquel, P. A. (2008). On physicalism and downward causation in developmental and cancer biology. Acta Biotheoretica, 56(4), 257-274.

Stoker, A. W., Hatier, C., \& Bissell, M. J. (1990). The embryonic environment strongly attenuates v-src oncogenesis in mesenchymal and epithelial tissues, but not in endothelia. Journal of Cell Biology, 111(1), 217-228.

Stylianopoulos, T. (2017). The solid mechanics of cancer and strategies for improved therapy. Journal of Biomechanical Engineering, 139(2), 021004.

Tomczak, K., Czerwińska, P., \& Wiznerowicz, M. (2015). The Cancer Genome Atlas (TCGA): An immeasurable source of knowledge. Contemporary Oncology,, $19(1 \mathrm{~A})$, A68.

Umerez, J., \& Mossio, M. (2013). Constraint. In W. Dubitzky, O. Wolkenhauer, K.-H. Cho, \& H. Yokota (Eds.), Encyclopedia of Systems Biology (pp. 490-493). New York, NY: Springer New York.

Vaux, D. L. (2011). In defense of the somatic mutation theory of cancer. BioEssays, 33(5), 341-343. 
Final draft of a chapter to be published in D. S. Brooks, J. DiFrisco \& W. C. Wimsatt (Eds.), Biological Levels:

Composition, Scale and Evolution in Complex Systems. MIT Press.

Weinberg, R. A. (2007). The biology of cancer (Vol. 1). New York: Garland Science.

West, A. K. V., Wullkopf, L., Christensen, A., Leijnse, N., Tarp, J. M., Mathiesen, J., ... \& Oddershede, L. B. (2017). Dynamics of cancerous tissue correlates with invasiveness. Scientific Reports, 7 , 43800 .

Wilson, M. (2012). What is classical mechanics anyway? In R. Batterman (Ed.), Oxford Handbook of Philosophy of Physics (pp. 43-106). Oxford: Oxford University Press.

Wimsatt, W. C. (1972). Complexity and organization. PSA: Proceedings of the Biennial Meeting of the Philosophy of Science Association, 20, 67-86.

Wimsatt, W. C. (1994). The ontology of complex systems: levels of organization, perspectives, and causal thickets. Canadian Journal of Philosophy, 20, 207-274.

Wimsatt, W. C. (2007). Re-engineering philosophy for limited beings: piecewise approximations to reality. Cambridge, MA: Harvard University Press.

Witz, I. P. (2009). The tumor microenvironment: the making of a paradigm. Cancer Microenvironment, 2(1), 9-17.

Woodward, J. (this issue). Downward caustion and levels. In D. S. Brooks, J. DiFrisco \& W. C. Wimsatt (Eds.), Biological Levels: Composition, Scale and Evolution in Complex Systems. MIT Press. 